\title{
Civilisations
}

Revue internationale d'anthropologie et de sciences

humaines

$41 \mid 1993$

Mélanges Pierre Salmon II

\section{Les structures d'enseignement et leur rôle dans l'histoire de l'Ethiopie impériale}

Alain Verhaagen

(2) OpenEdition

Journals

Édition électronique

URL : http://journals.openedition.org/civilisations/1737

DOI : $10.4000 /$ civilisations. 1737

ISSN : 2032-0442

Éditeur

Institut de sociologie de l'Université Libre de Bruxelles

Édition imprimée

Date de publication : 1 septembre 1993

Pagination : 459-481

ISBN : 2-87263-094-5

ISSN : 0009-8140

Référence électronique

Alain Verhaagen, « Les structures d'enseignement et leur rôle dans l'histoire de l'Ethiopie impériale », Civilisations [En ligne], 41 | 1993, mis en ligne le 30 juillet 2009, consulté le 19 avril 2019. URL : http:// journals.openedition.org/civilisations/1737 ; DOI : 10.4000/civilisations.1737

Ce document a été généré automatiquement le 19 avril 2019

(c) Tous droits réservés 


\title{
Les structures d'enseignement et leur rôle dans l'histoire de l'Ethiopie impériale
}

\author{
Alain Verhaagen
}

\section{Ménélik II, ou l'amorce d'une politique}

1 Ce fut avec la conversion au christianisme de l'empereur Ezana, vers 350 PCN, que s'annoncèrent les prémices d'une certaine éducation non formelle en Abyssinie ${ }^{1}$. En effet, dans la maison de lecture, en fait la cour de leur église, les prêtres commencèrent à inculquer aux jeunes enfants les caractères du guèze ${ }^{2}$, la lecture des textes sacrés et leur retranscription, conférant par là à leurs ouailles les connaissances fondamentales en matière de lecture et d'écriture. Ensuite, l'opportunité était offerte aux meilleurs éléments de persévérer dans différentes options : musique liturgique, poésie, théologie ou encore philosophie, au sein d'écoles dirigées par l'Eglise, telles les zema bet, quene bet ou masahaf bet. Néanmoins, au départ déjà, l'accès à la maison de lecture était considérablement restreint de facto dès lors que, parmi les familles d'agriculteurs, qui constituaient l'extrême majorité de la population ${ }^{3}$, rares étaient celles pouvant, raisonnablement, exempter, même ponctuellement, leurs enfants des travaux des champs. Les jeunes nobles, quant à eux, étaient envoyés directement dans les couvents, lesquels assureraient leur instruction ${ }^{4}$.

2 Au fil des siècles, l'Eglise allait de la sorte, en l'absence peu ou prou de toute initiative impériale, s'affirmer comme l'unique ${ }^{5}$ propagatrice du savoir, et ce jusqu'à la seconde moitié du dix-neuvième siècle, qui vit l'avènement d'un grand monarque. En effet, souverain éclairé, Ménélik II (1844-1913), qui fut sans nul doute, et reste, l'un des protagonistes les plus prestigieux de l'histoire éthiopienne dès lors qu'il fit pièce à l'invasion italienne, en 1896, et qu'il parvint à étendre considérablement les limites de l'empire, convint d'ouvrir l'Ethiopie au monde moderne, au vu, entre autres, de l'installation dans sa capitale, d'un nombre croissant de légations, hérauts d'une volonté 
des puissances européennes de conforter leurs relations avec un pays auquel, apparemment, entendre imposer la férule coloniale eût relevé de la chimère. Aussi fallaitil doter cette société médiévale d'une nouvelle composante apte à assimiler et développer les progrès techniques que les Occidentaux lui présentaient à l'envi'.

Ce fut ainsi que, au tout début du vingtième siècle, Ménélik II confia au kenyazmach Ibsa, dignitaire de la cour, la direction d'une école réservée à certains enfants de la noblesse et située dans l'enceinte même du palais ${ }^{7}$. Afin de ne pas s'attirer l'inimitié ouverte du clergé qui, désireux de préserver sa suprématie en matière d'éducation, dénigrait cette initiative, pour limitée qu'elle fût, Ménélik II sollicita, en 1906, la venue d'enseignants appartenant à la communauté copte d'Egypte ${ }^{8}$.

4 Finalement toutefois, faisant définitivement fi de l'opinion de l'Eglise, en octobre 1908, l'empereur inaugura l'Ecole impériale Ménélik II, premier établissement d'enseignement officiel', où étaient enseignés l'amharique, le français, l'anglais, l'italien, les mathématiques et les sciences. L'accès au collège était gratuit mais subordonné à la connaissance parlée et écrite de l'amharique, condition rédhibitoire pour les nonamarophones, ce qui limitait à nouveau à la seule noblesse l'accès à l'enseignement. Ce fut la même année encore que la première école provinciale ouvrit ses portes, à Harar, dans l'Est du pays ${ }^{10}$.

5 Parallèlement aux velléités impériales se développèrent, à la même époque, une kyrielle d'institutions privées et libres. Ainsi, en 1907, les Frères de Saint-Gabriel fondèrent, à Addis Ababa, une école pour jeunes autochtones, sous l'égide de la communauté française d'Ethiopie, laquelle en confia la direction à l'Alliance française, dès 1910. En 1912, une Ecole française fut créée dans la capitale, une institution similaire étant ouverte également à Dire Dawa, dans l'Est ${ }^{11}$. Globalement, à cette époque, une centaine d'écoles et collèges non officiels furent édifiés.

$6 \mathrm{Si}$, comme nous l'avons signifié, les structures naissantes d'enseignement se concentraient à Addis Ababa, il convient cependant de remarquer que, en Erythrée, dès la fin du dix-neuvième siècle, le colonisateur italien fit bénéficier ses troupes indigènes, les askaris $^{12}$, d'une instruction élémentaire (italien, arabe, amharique, arithmétique et géographie). Les religieux européens, et plus précisément les membres de la Mission évangélique suédoise ainsi que les Frères lazaristes, se dédiaient, eux, davantage à la mise en place d'institutions d'enseignement en faveur de toute la population de ce territoire (écoles traditionnelles, professionnelles et techniques). S'attirant par là l'animosité des Italiens, ils furent, hélas, expulsés par ceux-ci en 1894.

7 Cependant, malgré ce fâcheux avatar, la province de la $\mathrm{mer}^{13}$, forte des réalisations multiples des misssions religieuses suédoises et françaises, ne semblait, en ce début de vingtième siècle, rien avoir à envier à la capitale de l'empire en matière de structures d'enseignement.

8 Dès la fin de la première guerre mondiale, les différents ordres religieux occidentaux, vraisemblablement sollicités par leurs puissances tutélaires respectives, allaient se livrer à une compétition effrénée de prosélytisme dans l'enseignement. Ainsi, entre 1918 et 1924, lazaristes et capucins ouvrirent sept écoles secondaires ainsi qu'une trentaine d'établissements primaires ${ }^{14}$. D'autres écoles, toujours sous l'égide de l'Eglise catholique romaine, furent également créées à Harar, Dire Dawa et Sofi. L'Eglise luthérienne, par l'intermédiaire des missionnaires suédois ${ }^{15}$, administrait, quant à elle, onze écoles, dont une majorité implantées à Addis Ababa. L'Eglise adventiste du septième jour patronnait deux 
établissements ${ }^{16}$ et, enfin, l'Eglise presbytérienne unifiée d'Amérique du nord établit, elle, une école à Sayo.

9 L'enseignement dispensé dans ces institutions était généralement gratuit et recouvrait à peu près les mêmes curricula qu'en Europe, les langues d'enseignement étant, par ailleurs, tantôt l'anglais, le français ou l'amharique, tantôt une combinaison de deux d'entre elles ou des trois ${ }^{17}$.

10 Ménélik II allait donc léguer ainsi à ses successeurs les fondements d'un système éducatif largement conçu à l'aune occidentale et qu'il leur reviendrait de développer face aux défis futurs qu'avait présagés ce gouvernant averti.

\section{Haile Sellassie I, ou l'éducation au service du pouvoir}

\section{De Tafari Makonnen School à University College of Addis Ababa}

11 Ras Tafari Makonnen, régent depuis 1917, à la suite d'une des sempiternelles périodes de troubles qui ont ébranlé la dynastie salomonide depuis la nuit des temps, et qui a provoqué la chute de Lij Yasu, lequel a furtivement succédé à Ménélik II, mort en 1913, va poursuivre la politique d'éducation lancée par le père de l'Ethiopie moderne.

Inaugurant, en 1927, l'école Tafari Makonnen, destinée à remplacer l'ancienne Ménélik II, le régent souligne, dans son allocution, la corrélation entre éducation et indépendance, le progrès, et donc l'indépendance, étant subordonnés à l'enseignement. Et, finançant Tafari Makonnen de ses propres deniers, l'héritier du trône a ébauché la voie à suivre ${ }^{18}$, c'est au peuple qu'il incombe dès lors de persévérer] Les cours (français, anglais, anglais, arabe, arithmétique, chimie, physique) inclus dans le curriculum de cette nouvelle école sont dispensés par des professeurs français et libanais francophones, dont le traitement sera, en partie, garanti, dès 1926, par le prélèvement d'une taxe (6\% ad valorem) sur toute opération d'import-export. Il s'agit là de la première mesure de financement du secteur de l'éducation par les pouvoirs publics ${ }^{19}$.

13 La même année encore, le pouvoir impérial crée, cette fois, une école pour esclaves affranchis, et, de 1928 à 1929, l'école Saint-Georges $(\mathrm{F})^{20}$ ainsi que différents établissements provinciaux $^{21}$. Toutefois, éternelle pierre d'achoppement, seule la noblesse a les moyens d'y envoyer ses fils.

L'année de son intronisation (1930), l'empereur, qui a pris le nom d'Haile Sellassie I ${ }^{22}$, institue le ministère de l'Education et des Beaux-arts, lequel se voit allouer, en sus du produit de la taxe imposée depuis 1926, deux pour cent des revenus du Trésor ${ }^{23}$. Au début des années trente, huit établissements d'enseignement officiel sont ouverts en province et sept à Addis Ababa, dont le Lycée Haile Sellassie I (F) ${ }^{24}$. Et c'est en 1931 qu'apparaît la première institution d'enseignement pour filles de la noblesse, Itegue Menen, du nom de l'impératrice, qui en a promû la création. Parallèlement est fondée Medane Alem, école destinée à accueillir les enfants des balabat, chefs locaux des marches méridionales de l'empire.

15 Pendant quelques années encore, le pouvoir accroît le nombre d'établissements scolaires ${ }^{25}$, fondant également, en 1935, une école pour les enfants des ressortissants grecs, arméniens et indiens. En outre, il accorde des bourses d'études pour l'étranger, envoyant quelques centaines d'étudiants dans plusieurs pays du Proche-Orient, de même qu'en Europe occidentale et aux Etats-Unis. 
16 L'invasion italienne de 1935 va toutefois mettre à bas les projets impériaux. En fait, depuis 1907 déjà, en Erythrée, l'occupant a décrété le développement séparé, qui porte en germe le ferment de la politique dévastatrice que feront leur, à partir de 1922, les maitres du futur et éphémère empire d'Afrique orientale. Aussi, dès l'occupation d'Addis Ababa, en mai 1936, les Italiens excluent-ils virtuellement les autochtones de l'enseignement secondaire, ferment nombre d'écoles officielles, expulsent quasi tous les missionnaires étrangers, au premier rang desquels les protestants, et l'ordre mussolinien s'installe pour cinq ans.

Après la libération du pays, en mai 1941, le pouvoir semble entendre doter le secteur de l'enseignement de nouveaux moyens, instituant, en 1947, une taxe foncière qui servira à financer les activités du ministère de l'Education nationale, et portant son budget de un million de dollars éthiopiens ${ }^{26}$ en 1943, à dix millions en 1950. Cette majoration se révèle d'autant plus nécessaire que, en ce début des années cinquante, il est sérieusement envisagé de jeter les bases d'un enseignement supérieur alors que, jusqu'à présent, seul un séjour à l'étranger permettait de s'affranchir du niveau de l'enseignement secondaire.

Ainsi, en décembre 1950, la première institution d'enseignement supérieur, le University College of Addis Ababa, dirigée par des jésuites canadiens, ouvre ses portes, accueillant 220 étudiants. Dans le but de diversifier un enseignement à caractère fort général au départ, il est vrai, le University College se voit adjoindre, en quelques années, différents départements : ingénierie (1953), génie civil (1954), agronomie (1954) et santé publique (1954). Tout aussi intéressante est la création, dès 1953, du University College Extension Service, système original s'adressant aux Ethiopiens appartenant à la classe moyenne de la population active et désireux d'acquérir une formation complémentaire tout en poursuivant leurs activités professionnelles. Il s'agit de cours de fin de journée, étalés sur sept ou huit années et conférant trois niveaux différents de diplôme ${ }^{27}$. D'emblée, cette seule section enregistre 150 inscriptions en 1953, et déjà 367 trois ans plus tard ${ }^{28}$.

Partant, en cette fin de décennie, l'avenir de l'enseignement semble, apparemment, se dessiner sous de favorables auspices et, a fortiori, lorsque, en 1959, une mission universitaire américaine de l'Etat d'Utah met la dernière main au projet de fondation de l' Haile Sellassie I University, dont un comité ministériel a esquissé l'idée depuis 1951.

\section{De la Conférence d'Addis Ababa au projet Unesco-PNUD}

En 1961, l'empereur, qui, en décembre de l'année précédente, a failli être renversé par un soulèvement de la garde, inaugure la Conférence des ministres de l'Education des Etats africains. La conférence d'Addis Ababa vise, d'une part, à proposer un plan d'africanisation des systèmes hérités de la colonisation et, d'autre part, à inventorier les carences existantes ainsi que les adjuvants nécessaires à l'essor du secteur.

Et, stupéfaction, un des principaux documents de travail ${ }^{29}$ jette l'anathème sur l'Ethiopie ! En effet, les synopsis statistiques renvoient invariablement l'empire au rang des laisséspour-compte. L'Ethiopie, symbole, égérie même, de ces Etats, aurait-elle failli, envers ses sujets, à un des premiers devoirs que, précisément, entendent s'assigner ses jeunes pairs? Pourquoi?

Avant d'aborder le fondement de cette situation particulièrement délétère, il conviendrait de se référer brièvement à quelques éléments statistiques caractéristiques de l'année scolaire 1960-1961 $1^{30}$. Ainsi, pour l'enseignement primaire, le nombre total des inscriptions ne représente que 5,6\% de la classe d'âge des degrés (grade) 1 à $8^{31}$, à savoir 
quatre millions d'élèves potentiels; pour l'enseignement secondaire, $1 \%$ de la classe d'âge des degrés 9 à 12 , soit environ deux millions et demi d'élèves potentiels ${ }^{32}$; quant à l'enseignement supérieur, le pourcentage est insignifiant. En ce qui concerne les écoles, 1019 ont été ouvertes dans le primaire et 46 dans le secondaire. Toutefois, sur les 1019 premières, $30 \%$ seulement dispensent un enseignement jusqu'au degré 6 , et, de ces $30 \%$, uniquement les deux tiers assurent un enseignement jusqu'au degré 8. Pour le secondaire, seuls 28 des 46 établissements sont officiels; de ces 28,14 sont localisés à Addis Ababa et regroupent $40 \%$ de toutes les inscriptions du pays pour les degrés 9 à 12 , avec même 60 \% de toutes les inscriptions du pays pour le degré $12^{33}$.

Mais ce n'est pas à l'aune des critères d'évaluation de la situation prévalant dans d'autres pays africains que doit être approché le problème de l'éducation en Ethiopie, en cette année 1961, en ce sens que ce sont les réalités profondes de la société elle-même qui recèlent en leur sein la logique causale de cette situation, légitimant, somme toute, tout à fait les options du pouvoir.

En fait, la structure pyramidale de la société est dominée par un seul homme, l'empereur, monarque absolu. Toutefois, depuis les temps immémoriaux, constants ont été les accès des éditions des grands feudataires et les intrigues de palais précipitant l'accession au trône d'un nouveau prince amhara ${ }^{34}$. En sus, depuis la fin du dix-neuvième siècle, l'Abyssinie s'est considérablement étendue du fait des conquêtes de Ménélik II, et ces marches de l'empire que forment les nouvelles provinces lointaines ne sont pas encore totalement colonisées. C'est pourquoi, dès son avènement, et même avant, Haile Sellassie I n'aura de cesse d'oeuvrer pour la consolidation de son pouvoir et la prééminence de la culture amhara. Et ce par le biais d'une administration hypercentralisée, sinon totalement amhara au moins amharophile, dont l'allégence sera indéfectiblement garantie au roi des rois dès lors que c'est à lui, initiateur du réseau d'enseignement qui la générera, qu'elle devra son existence et ses privilèges, principalement fonciers, lesquels ne peuvent être concédés que par l'empereur, propriétaire, de droit divin, de toutes les terres du pays. Aussi, dès 1917, comme nous l'avons vu déjà, celui qui n'est encore que le régent de l'empire va entreprendre le développement de son système d'éducation.

En aval des institutions d'enseignement se situent le University College et la future Haile Sellassie I University, dont les épreuves éliminatoires favorisent notoirement les candidats de l'ethnie dominante, consentant toutefois à des étudiants non-amharas de conclure brillamment leurs études, non par condescendance, mais dans l'idée qu'ils constitueront plus tard les mandataires utiles de l'autorité impériale auprès des peuplades colonisées, dès lors que, étant des leurs, ils s'y intégreront moins difficilement. Il y a lieu de remarquer cependant que les cols-blancs non-amharas n'accéderont, en règle générale, que sporadiquement à des responsabilités supérieures; ainsi, par exemple, sur les 119 portefeuilles ministériels attribués de 1944 à 1966, 96 écherront à des Amharas, dont 85 à des Shoans ${ }^{35}$.

En amont se trouve le réseau des écoles secondaires et primaires, dont il n'y a pas lieu d'accroître le nombre puisque, si les effectifs sont limités dorénavant à quelque 250.000 inscrits dans le primaire et le secondaire, moins d'un pour cent auront, vraisemblablement, accès, plus tard, à l'enseignement supérieur, ce qui suffira à assurer la relève dans l'administration et, partant, la pérennité du système.

Quant à la langue d'enseignement, cela ne souffre aucun doute, ce sera exclusivement l'amharique, prédisposant naturellement donc la jeunesse amhara. 
28 En ce qui concerne l'éducation non formelle, rien, ou presque, n'a été entrepris jusqu'à présent en matière d'alphabétisation des adultes, le pouvoir considérant que la seule finalité de celle-ci est d'inciter les néo-alphabètes à s'insérer par la suite dans le système formel afin d'accéder à une strate sociale supérieure, ce qui, à terme, déséquilibrerait la société. En 1955 toutefois, l'empereur, dans sa Proclamation sur l'alphabétisation, a annoncé la mise sur pied imminente d'une Armée de l'alphabet ${ }^{36}$. Mais, d'une part, la campagne devrait être conduite dans une seule langue, l'amharique ${ }^{37}$, et, d'autre part, aucune mesure adéquate ne semble avoir été arrêtée pour son financement ${ }^{38}$.

En réalité, la conférence d'Addis Ababa, qui a jeté la lumière sur cet inquiétant tableau, va, loin de mettre l'empire au ban des autres nations, susciter une attention sans cesse croissante de la part des Occidentaux à l'endroit du développement de l'éducation en Ethiopie. C'est en cette année 1961, d'ailleurs, que l'Université Haile Sellassie I vient d'être inaugurée, accueillant 948 étudiants au sein de sept facultés, académies et instituts: Beaux-arts, sciences, technologie, agronomie, génie civil, santé publique et théologie. La même année encore, une école sociale y est annexée. En 1962, d'une part, les Etats-Unis mettent sur pied la faculté de psycho-pédagogie, fournissant enseignants et matériel pédagogique, et, d'autre part, le système de cours de promotion sociale, le University college extension service, créé en 1953, est incorporé à l'université, devenant la University extension division. En 1962 toujours, mais cette fois dans le cadre de l'éducation non formelle, qui, en fait, est encore embryonnaire, le roi des rois institue, sous son autorité directe, l'Organisation de la campagne nationale d'alphabétisation, dont la présidence échet au prince héritier. Cet été-là, 13.280 personnes suivront d'ailleurs les cours d'alphabétisation.

Il semble donc que, au lendemain du réquisitoire sentencieux du document de l'Unesco, les gouvernants fouaillent toutes les énergies nationales et étrangères pour démentir les conjectures de 1961. Et c'est dans ce contexte que, en 1962, la mission exploratoire menée par l'Unesco recommandera à l'Agence Internationale pour le développement ${ }^{39}$ l'octroi d'un premier crédit à l'Ethiopie ${ }^{40}$. En 1963, ce sont les onzième et douzième dépendances de l'université qui sont inaugurées, à savoir la faculté de droit et l'école de gestion ${ }^{41}$. Un an plus tard, la Congrégation catholique Piae Madre Nigritiae crée, en Erythrée, l'université d'Asmara, seule institution non gouvernementale jamais financée dans l'enseignement supérieur. Et, parallèlement, commencent à se multiplier les écoles privées et libres dans l'enseignement primaire et secondaire.

31 C'est en 1964 également que les étudiants de l'université Haile Sellassie I obtiennent la création de l'Ethiopian University service. Cette revendication, toute empreinte de civisme, vise à inclure dans le programme d'un cycle universitaire une année d'assistance directe assurée par les étudiants au sein d'une communauté rurale ${ }^{42}$. Cette initiative estudiantine dénote manifestement une prise de conscience, par la nouvelle génération d'intellectuels, de l'anachronisme qui caractérise l'Ethiopie féodale, et qui ne pourra, raisonnablement, se perpétuer indéfiniment ${ }^{43}$. Toutefois le pouvoir impérial ne semble pas faire grand cas de ce malaise diffus qui commence à pénétrer l'élite de demain.

Les années soixante sont encore marquées par la création d'un département de recherche, planification et évaluation au sein du ministère de l'Education et des Beauxarts. En réalité, une initiative identique avait déjà vu le jour dans les années cinquante mais, vite jugée inintéressante sans doute, elle avait détourné d'elle toute prodigalité impériale. Cette fois, un vice-ministre est nommé à la tête de ce département dont l'effectif, fort seulement de quelques fonctionnaires, va, hélàs, devoir se disperser 
considérablement au vu du nombre de ses attributions: curricula, matériel didactique, planification, statistiques, mass media, organisation des examens, recherche et publication.

En 1968, le gouvernement adhère au Programme mondial d'alphabétisation ${ }^{44}$ lancé par l'Unesco et le PNUD, et dont la FAO ainsi que l'OIT prendront en charge les formations agricole et professionnelle. Le fonds spécial du PNUD assurera le financement du projet à concurrence de 1,5 million US \$, auquel s'ajouteront 2,5 millions US \$ sous forme de matériel fourni par le gouvernement. Conformément aux prescriptions de l'Unesco, les experts commis au PAAOP ${ }^{45}$ sélectionnent ainsi des micro-environnements spécifiques, où, conformément au nouveau concept d'alphabétisation fonctionnelle, il sera possible d'évaluer l'incidence d'une symbiose réalisée entre l'alphabétisation et les activités menées traditionnellement en ces sites ${ }^{46}$. Il s'agit là en réalité d'une optique novatrice de l'Unesco dès lors que, jusqu'au milieu des années soixante, l'organisation internationale a privilégié l'aspect scolaire de l'alphabétisation. Toutefois, considérant le coût rhédibitoire, pour les pays moins développés, d'une telle entreprise visant exclusivement à l'apprentissage de la lecture et de l'écriture, l'Unesco entend désormais coordonner curricula des campagnes d'alphabétisation et développement socio-économique des pays concernés, dans le but de rentabiliser son action, tout en lui préservant sa dimension de promotion de la dignité humaine. L'alphabétisation fonctionnelle implique donc la préparation de curricula et d'un matériel didactique en étroite relation avec les activités quotidiennes des 100.000 futurs apprenants prévus. C'est ainsi qu'abécédaires, textes de suivi, films d'animations et émissions radiophoniques mis au point pour ce projet, qui devrait s'échelonner jusqu'en 1973, se concentreront sur des thèmes afférents, tels l'agriculture, le foyer, l'artisanat rural, la manufacture ou encore l'éducation sanitaire.

Ces années soixante s'achèvent, en fait, sur un constat à tout le moins ambigu. En effet, d'une part, les révélations de la conférence d'Addis Ababa pourraient incliner à penser que les gouvernants éthiopiens, aidés par la communauté internationale certes, mettront tout en oeuvre pour que leur pays recouvre le prestige considérable dont il jouissait auprès de ses pairs africains, et le vaste projet PAAOP ainsi que l'essor de l'université Haile Sellassie I sont peut-être les premiers signes d'une telle nouvelle détermination. D'autre part cependant, et paradoxalement a priori puisqu'ils semblent désormais attirer la meilleure attention des autorités tutélaires, les étudiants contestent de plus en plus ouvertement ces mêmes gouvernants, mais en dépassant largement le cadre des traditionnelles revendications puisque, aussi bien, ils remettent en cause les fondements de la société éthiopienne. L'interrogation se pose dès lors de savoir si, en cette fin de décennie, les nouvelles dispositions arrêtées par les gouvernants s'inscrivent réellement dans une stratégie globale de développement du système éducatif ou bien s'il ne s'agit là que d'un cautère sur une jambe de bois, appliqué par un pouvoir refusant de sentir que, déjà, peut-être, ses bases vacillent dangereusement.

\section{4 : bilan d'un anachronisme}

\section{Le système non formel}

Instituée en 1962, l'Organisation de la campagne nationale d'alphabétisation a mené l'alphabétisation de 19.889 personnes, en 1963, et de 26.977 autres en 1964. Toutefois, financée exclusivement par des contributions privées et animée par des alphabétiseurs 
tous volontaires, la structure périclite bientôt inéluctablement, n'assurant plus, en 1969, que l'alphabétisation de 7.500 adultes, avant de tomber en totale déliquescence dès le début des années soixante-dix ${ }^{47}$. De la sorte, si le directeur général de l'éducation pour adultes peut avancer, triomphalement, que, de 1963 à 1974, 1.700.000 Ethiopiens ont été alphabétisés, il conviendrait de garder à l'esprit que, d'une part, ce résultat est à mettre au crédit des efforts consentis par les étudiants, les enseignants, l'Eglise et les missions qui, sans discontinuer, se sont dédiés à cette tâche, et que, d'autre part, la population étant passée de 22 à 26 millions d'âmes, le nombre de néo-alphabètes a certes crû mais le fléau est loin d'être conjuré. Et, pourtant, la Commission pour la réforme de l'éducation, qui a siégé de 1971 à 1972, a proposé, dans son rapport final, l'alphabétisation annuelle de $5 \%$ de la classe d'âge 20-24 ans ${ }^{48}$ !

Quant au projet de l'Unesco qui se conclut en cette année 1974, s'il n'a pas dégagé un output quantitatif édifiant, avec 43.400 hommes et femmes alphabétisés en six ans, il a néanmoins permis d'identifier les besoins d'une population, de développer des méthodes appropriées d'alphabétisation fonctionnelle dans des environnements spécifiques, de mettre au point un matériel didactique au fait de ces mêmes environnements, de même qu'il a assuré la formation d'experts et d'alphabétiseurs autochtones, le tout représentant une expérience tout à fait digne d'intérêt ${ }^{49}$.

En bref, en 1974, l'analphabétisme afflige encore plus de $90 \%$ de la population globale, et l'absence totale d'initiative en faveur du monde rural confirme péremptoirement l'immobilisme originel de la société. En effet, si, en zone urbaine, $52 \%$ des hommes et $16 \%$ des femmes, de la tranche d'âge 10-65 ans et plus, sont alphabétisés, ce taux n'atteint que, respectivement, 9,5 et $0,5 \%$ en zone rurale ${ }^{50}$. Plus largement, sur une population totale de 26,6 millions d'âmes, 18,3 millions sont âgées de dix ans et plus. Et, si l'on considère que cette tranche de la population peut approximativement être répartie en $50 \%$ de femmes et $50 \%$ d'hommes, et que, respectivement, $6 \%$ des femmes et $13 \%$ des hommes sont alphabètes, cela donne un total d'environ 1.740.000 Ethiopiens alphabétisés pour la tranche d'âge 10-65 ans.

\section{Le système formel}

En 1961, à la lumière du Plan d'Addis Ababa, le ministre de l'Education nationale et des Beaux-arts admettait que, pour se conformer aux recommandations de ce dernier, l'Ethiopie eût dû octupler son budget national et consacrer entièrement celui-ci au seul développement de l'éducation. Convenons toutefois que les participants à la conférence de 1961 péchaient manifestement par excès d'enthousiasme dans leurs conclusions, escomptant au lendemain des indépendances la mise en oeuvre, par les anciennes puissances coloniales, d'une coopération disposée à sacrifier des sommes colossales sur l'autel de l'émancipation intellectuelle des peuples du Tiers monde. Certes, cependant il y a manifestement lieu d'entreprendre un effort immense et rapide en Ethiopie afin, sinon de combler à court terme le fossé séparant les deux pôles de la société, au moins d'enrayer cette involution du secteur de l'enseignement.

En données absolues, incontestablement, l'éducation nationale a engrangé des résultats positifs. Ainsi son budget est-il passé de 28 millions de dollars éthiopiens en 1962, à 56,6 millions en 1967, et à 96,6 millions en 1974 ; ce qui constitue une majoration de $245 \%$ en 12 ans $^{51}$. Toutefois la ville a, à nouveau, accaparé les investissements aux dépens du milieu 
rural. Ainsi, bien qu'elles ne regroupent que $10 \%$ de la population, les zones urbaines disposent de la moitié des infrastructures scolaires du pays ${ }^{52}$.

Le corps enseignant, quant à lui, est constitué à raison de $47 \%$ de professeurs étrangers, indiens principalement; et la plupart de ces expatriés sont directement rémunérés par l'aide occidentale. C'est d'ailleurs afin de pallier cette carence que, en 1972, la BIRD ${ }^{53}$ et l'AID ont financé la création de l'académie pédagogique de Bahir Dahr, l'assistance technique et le matériel étant garantis par l'Unesco et le PNUD. L'académie s'emploie à former des pédagogues éthiopiens qui, à leur tour, devront mettre sur pied un réseau d'écoles normales.

41 Par ailleurs, les termes relatifs de l'évolution du secteur de l'enseignement dans le primaire et le secondaire ${ }^{54}$, mettent en évidence la modicité des moyens mis en oeuvre, par rapport aux besoins d'une population dont le rythme annuel de croissance avoisine $3 \%{ }^{55}$. En outre, la multiplication des écoles privées, 1.283 sur les 3.287 inventoriées pour l'année 1973-1974 ${ }^{56}$, confirme cette faiblesse, beaucoup plus structurelle que conjoncturelle, nous l'avons déjà noté. La conclusion en est que "(...) le pourcentage des enfants en âge d'aller à l'école primaire et qui y [vont] effectivement ne dépass[e] pas $18 \%$; le pourcentage correspondant [est] de $8 \%$ environ pour le premier cycle de l'enseignement secondaire et de $3 \%$ environ pour le deuxième cycle. Le taux de scolarisation [est] encore plus faible dans les zones rurales, très souvent inférieur à $1 \%$ des enfants d'âge scolaire" ${ }^{157}$.

42 Mais, en ce début d'année 1974, ce sont, cette fois, les enseignants éthiopiens, qui, à leur tour, entrent en lice. En fait, ceux-ci veulent s'opposer à une réforme du système d'éducation prônée par les experts internationaux, lesquels, entre autres, soulignent la nécessité de généraliser l'enseignement primaire. Or, si cette réforme aboutit, beaucoup d'entre eux devront quitter Addis Ababa pour l'intérieur du pays, perspective qu'ils refusent catégoriquement.

Enfin, dernier niveau de l'éducation formelle, l'enseignement supérieur est agité, depuis quelques années, de soubresauts préoccupants. En effet, il convient de se rappeler que, en 1964, les étudiants ont réclamé l'institutionnalisation de l'ethiopian university service, initiative qui trahit la préoccupation d'aucuns quant aux conditions de vie de l'écrasante majorité de la population. Un an plus tard, ils ont manifesté pour la proclamation d'une réforme agraire, remettant par là en question le mode de tenure foncière dont ils savent pertinemment qu'elle constitue l'instrument essentiel de la pérennité de la société et, partant, de ce pouvoir. En réalité donc, il ne s'agit pas tant de vilipender le régime pour la qualité de l'enseignement, bien que, en termes relatifs, les crédits aient diminué de moitié, entre 1970 et 1973, vu l'accroissement du nombre d'étudiants, soit 1.000 inscrits en 1962-1963 et plus de 4.000 en 1969-1970. Le sujet d'inquiétude est plutôt l'insuffisance de débouchés professionnels dans un secteur secondaire pratiquement inexistant, alors que la fonction publique, elle, est déjà pléthorique.

La contestation estudiantine s'est intensifiée, aboutissant, en 1969, à l'assassinat, par les forces de l'ordre vraisemblablement, du président de l'association des étudiants, ce qui a déclenché une vague d'agitations sur le campus, suivie d'une répression violente de la garde impériale qui a fait dix morts et cent blessés ${ }^{58}$. Deux ans plus tard, mille étudiants ont été confinés dans divers camps de travail, et nombre de leurs condisciples quittaient délibérément l'université, en signe de protestation ${ }^{59}$. 

appeler outre mesure l'attention si, toutefois, elle ne s'inscrivait pas directement dans un contexte plus général particulièrement délétère. En effet, çà et là dans les villes, couvent des troubles civils en raison de la terrible famine sévissant dans les provinces septentrionales, laquelle engendre une pression croissante sur ces mêmes centres urbains ${ }^{60}$. En concomitance, et en partie conséquemment, l'armée remet de plus en plus en cause l'autorité impériale ${ }^{61}$. Aussi, en quelques mois, cette dernière va-t-elle tomber de Charybde en Scylla, pour entraîner finalement dans une dérive fatale la plus vieille dynastie de la planète.

C'est ainsi que, le 12 septembre 1974, après avoir battu en brèche, pan après pan, le pouvoir du suzerain, le Comité de coordination des forces armées, de la police et de la garde nationale ${ }^{62}$ dépose l'empereur, dissout le parlement, suspend la constitution et institue un Conseil militaire administratif provisoire ${ }^{63}$, qui assumera le pouvoir. En moins d'un an donc, le cours d'une histoire plurimillénaire a basculé précipitamment dans une révolution radicale aux arcanes complexes, qui, en cet automne 1974, laisse croire en l'avènement d'une ère nouvelle pour l'Ethiopie et dont, pour quelques mois encore, le monde de l'enseignement va demeurer un des principaux hérauts.

\section{NOTES}

1. Les habitants de la péninsule yéménite entretenaient, depuis l'antiquité, des relations commerciales suivies avec une peuplade occupant l'autre rive du Bâb al-Mandab, les Habascha. Et, par extension, ils dénommèrent ainsi toute la région. C'est ainsi que, aujourd'hui encore, Habascha signifie Ethiopie en arabe classique. Les géographes européens, se fondant sur cette appellation, désignèrent dès lors du nom d'Abyssinie le plateau granitique situé à l'est de l'Afrique, entre la mer Rouge et le Nil.

2. Le guèze est une des langues de la troisième (sémitique) branche d'une (afro-asiatique) des quatre familles qui se partagent l'Afrique. issu du guèze, l'amharique, langue de l'ethnie au pouvoir jusqu'en 1974, les Amharas, fut consacré langue officielle du pays par la constitution révisée de 1955. Supplanté par l'amharique comme langue nationale, le guèze allait néanmoins demeurer la langue liturgique de l'Eglise copte ethiopienne.

3. En 1988, les agriculteurs constituaient encore $88 \%$ de la population totale (Cfr. Rapport mondial sur le développement humain 1990, New York, Programme des Nations Unies pour le développement (PNUD), 1990, p. 154).

4. Cfr. PANKHURST, R., "Education Language and History : An Historical Background to Post-War Ethiopia", The Ethiopian journal of Education, Vol. VII, n 1, June 1974, pp. 75-77.

5. Certes existait également un réseau d'écoles coraniques, mais beaucoup moins nombreuses, et dont le curriculum d'enseignement se limitait à l'apprentissage et l'interprétation des textes sacrés.

6. Cfr. SEDOC ETHIOPIA, L'Ethiopie aujourd'hui. Aspects du développement, Addis Ababa, French Embassy, Service of Documentation and Communication for Development, novembre 1977, p. 116 : "Ménélik eut à faire face à un phénomène complètement nouveau pour un empereur éthiopien : le besoin d'une élite éduquée pour faire face à l'ouverture au monde moderne de 
l'Ethiopie. Addis Abeba était remplie de légations étrangères déterminées à signer des traités, fixer des frontières, établir des relations commerciales et obtenir des concessions ; mais aussi de marchands, agents, aventuriers, cherchant à obtenir des contrats, franchises et emplois comme conseillers, interprètes, artisans, mercenaires etc...".

7. Cfr. ROSEN, E., Eine deutsche Gesandtschaft in Abyssinien, Leipzig, 1907, p. 267.

8. En vertu d'un accord historique signé en 1300, l'abouna, métropolite de l'Eglise copte (monophysite) d'Ethiopie, ne pouvait être éthiopien, de même était-il investi par le patriarche d'Alexandrie. Cette situation prévalut jusqu'à la fin de la première moitié du vingtième siècle.

9. Afin de faciliter l'appréhension du système d'éducation, nous appliquerons, en ce qui concerne les réseaux d'enseignement, la terminologie en vigueur en Belgique. Ainsi distinguerons-nous les écoles officielles, ou gouvernementales (governmental), des écoles libres ( church's) et privées (public).

10. Cfr. PANKHURST, E.S., Ethiopia. A cultural History, Woodford Green, 1955, pp. 534-535.

11. Cfr. JONES, T.J., Education in East Africa, London, s.d., p. 327.

12. En arabe classique, askari signifie gardien ou homme d'armes. Aussi les explorateurs européens, partis de la côte orientale de l'Afrique, dénommèrent-ils askari les Noirs enrôlés pour protéger leurs caravanes. Par extension, ce nom fut donné, plus tard, aux soldats des troupes d'infanterie indigène recrutés parmi les populations musulmanes d'Afrique et d'Asie.

13. L'empereur Zara Jacob, qui était monté sur le trône en 1434, avait divisé l'empire en unités administratives. C'était ainsi que la province de la mer - Erythrée étant une dénomination purement européenne - était dirigée par le roi de la mer, le bahar nagash.

14. A Alitena, Gula et Addis Alem.

15. Mission suédoise évangélique et Mission suédoise des amis de la bible.

16. A Addis Ababa et Addis Alem.

17. Cfr. PANKHURST, R., op.cit., p. 89.

18. Plus exactement, le régent suivait la ligne de Ménélik II dont le trésor impérial avait financé la mise sur pied et le fonctionnement de la première école officielle (Cfr. SEDOC ETHIOPIA, op.cit., p. 116).

19. Cfr. PANKHURST, R., op.cit., p. 90.

20. $(\mathrm{F})$ = le français comme langue d'enseignement.

(E) = l'anglais comme langue d'enseignement.

21. A. Dessie (F), Dire Dawa (F-E), Djidjiga (E) et Lekempt (E).

22. Haile Sellassie signifie force de la Sainte-Trinité enamharique.

23. Cfr. PANKHURST, R., op.cit., p. 91.

24. Rebaptisé Lycée franco-éthiopien à la révolution de 1974.

25. A Harar (F), Ambo (F), Djimma (F), Salale (F), Asba Tafari (E), Gondar (E), Debre Markos (E), Adwa (E) et Makale (E).

26. Rebaptisé birr le21 septembre 1976, la devise éthiopienne vaut 0,4825 US \$.

27. Certificate, diploma, degree.

28. Cfr. GOVERNMENT OF SOCIALIST ETHIOPIA, Twenty Years of Experience in the Reform, Redirection and Expension of Educational Services. The Case of Ethiopia, Country paper prepared for MINEDAF 5, The Conference of Ministers of Education and those responsible for Economic Planning of African member States, on Educational Policy and Cooperation, Harare, Zimbabwe, 28 June to 3 July 1982, Addis Ababa, Ministry of Education, May 1982, pp. 42-43.

29. Cfr. The Educational Situation in Africa Today, Conference Document EDAF/s/', 1961.

30. L'Ethiopie utilise le calendrier julien. L'année commence le 11 septembre (maskarem 1) et comprend douze mois de 30 jours ainsi qu'un mois de 5 ou 6 jours (pagume), selon que l'année est bissextile ou non. Notre année scolaire 1960-1961 correspond donc à l'année scolaire éthiopienne 1953. Nous utiliserons ici le calendrier grégorien. 
31. L'enseignement primaire et secondaire étant, à l'époque, réparti en, respectivement, 8 et 4 années, nous respecterons cette distinction. En 1967, la structure sera modifiée comme suit: niveau primaire (primary): degrés 1 à 6 ; niveau secondaire, cycle inférieur (junior secondary) : degrés 7 et 8 ; niveau secondaire, cycle supérieur (senior secondary) : degrés 9 à 12 .

32. Cfr. GOVERNMENT OF SOCIALIST ETHIOPIA, Twenty Years.., op.cit., p. 5.

33. Cfr. ibid., p. 6.

34. En arabe classique, ahmar qualifie un individu métissé et l'analogie avec le teint généralement clair de l'ethnie ahmara semble plausible. Depuis le quatorzième siècle, comme le relate le Kebra Nagast (ce Livre des rois est une chronique compilée par les moines éthiopiens depuis cette époque), les Ahmaras de la province du Shoa dominent l'empire.

35. Cfr. VANDERLINDEN, J., L'Ethiopie et ses populations, Bruxelles, Complexe, 1977.

36. Yefidal serawit, en amharique.

37. Cfr. THE DEPARTMENT OF ADULT EDUCATION, General Adult Education Programmes in Ethiopia with Emphasis on the National Literacy Campaign, Addis Ababa, Ministry of Education, September 1980, p. 2 : "In a country where about ninety languages are spoken, fourteen of which are widely spoken the official language, Amharic, was the language used as the only medium of instruction, both in the formal and non formal education".

38. Cfr. COMITE DE COORDINATION DE LA CAMPAGNE NATIONALE D'ALPHABETISATION, Chaque Ethiopien saura lire et écrire pour toujours !, Addis Ababa, mai 1981, pp. 8-10.

39. AID, groupe de la Banque mondiale.

40. Cfr. GOVERNMENT OF SOCIALIST ETHIOPIA, Twenty Years..., op.cit., p. 9 : "Capital funds were provided through the first and second IDA Credit Programmes which began in February 1966, for the purpose of equiping schools in which students were already enrolled".

41. En 1966, avec l'ouverture de la faculté de médecine, l'extension de l'université se clôturera.

42. Le système restera en vigueur jusqu'en 1970-1971.

43. Cfr. GOVERNMENT OF SOCIALIST ETHIOPIA, Twenty Years..., op.cit., pp. 44-46.

44. Pour l'Ethiopie, ce sera un Projet d'alphabétisation des adultes à orientation professionnelle, PAAOP.

45. Pour l'ensemble des données et enseignements du PAAOP Ethiopie, Cfr. Functional Literacy in Ethiopia, Imperial Ethiopian Government/UNDP/UNESCO Work-Oriented-Adult-Literacy-Project, Addis Ababa, Ministry of Education and Fine Arts, 1974, 75 pages, 6 annexes; SINGH, B.N. \& EVALUATION AND RESEARCH SECTION, IMPERIAL ETHIOPIAN GOVERNMENT/UNDP/UNESCO Work Oriented Adulte Literacy Pilot Project - Ethiopia - Final Evaluation Report, Addis Ababa, Ministry of Education and Fine Arts, December 1973.

46. Quatre zones d'action furent retenues: le centre de développement agricole de Wollamo (projet du gouvernement et de la Banque mondiale dans la région de Sidamo, le centre de développement agricole de Chilalo (projet de l'Agence suédoise internationale de développement - SIDA - dans l'Arssi), les caféières de Djimma et le complexe industriel en développement le long de l'axe Addis Ababa - Metahara.

47. Cfr. COMITE DE COORDINATION DE LA CAMPAGNE NATIONALE D'ALPHABETISATION, op.cit., p. 11.

48. Ibid., p. 13.

49. Ibid., p. 12.

50. Cfr. SEDOC ETHIOPIA, L'Ethiopie...,op.cit., p. 118.

51. Cfr. GOVERNMENT OF SOCIALIST ETHIOPIA, Twenty Years..., op.cit., pp. 7 et 12.

52. Cfr. THE DEPARTMENT OF ADULT EDUCATION, General Adult..., op.cit., p. 2.

53. Banque internationale pour la reconstruction et le développement, groupe de la Banque mondiale.

54. Cfr. MINISTRY OF EDUCATION, PLANNING SERVICES, 2974 E.C. (1981-1982) School Year - Mid Year Educational Statistics, Addis Ababa, Ministry of Education, August 1974 E.C. (1981/1982), pp. 1-2. 
55. Cfr. LEFORT, R., Ethiopie : la révolution hérétique, Paris, François Maspero, Cahiers libres, 362, 1981, p. 46 : "Entre 1956 et 1971, les effectifs augmentaient annuellement de $11 \%$ dans le primaire [et] de $19 \%$ dans le secondaire (...). Or l'Etat démontrait son incapacité à ajuster les moyens aux besoins liés à cette croissance. Ainsi, les dépenses annuelles (...) pour chaque élève du primaire (...) déclinèrent de 145 dollars en 1955 à 86 en 1965, chiffre qui continua à baisser par la suite".

56. Cfr. MINISTRY OF EDUCATION, PLANNING SERVICES, 1974 E.C (1981-1982) School Year..., op.cit., p. 3.

57. GUDETA MAMMO, "Ethiopie : la campagne nationale d'alphabétisation", Perspectives, Revue trimestrielle de l'éducation, vol. XII, $\mathrm{n}^{\circ} 2,1982$, p. 206.

58. Cfr. LEFORT, R., op.cit., p. 45.

59. Cfr. GOVERNMENT OF SOCIALIST ETHIOPIA, Twenty Years..., op.cit., p. 44 : "In the Academic year 1971-72, out of a total enrolment of 4757 in all faculties and colleges, 2147 ( $45 \%$ of the student body) voluntarily withdraw from the university in protest".

60. Cfr. LEFORT, R., op.cit., p. 67 : "La famine dans le Wollo et le Tigré ne mit pas le feu dans les campagnes mais rejaillit dans les villes, sous la forme d'une flambée des prix alimentaires - avec toutes ses conséquences sociales - et d'une vague d'indignation dans les secteurs les plus éclairés ; d'où une agitation fébrile sur le campus et dans les lycées".

61. Cfr. Ibid., pp. 80-81 : "Enfin, depuis la fin de l'année 1973, se multiplient au sein de l'armée des "comités du pot" qui rédigent des cahiers de doléance. On ne se rend pas alors compte que derrière ce mouvement strictement corporatiste, appuyé en sous-main par les officiers supérieurs (...), s'ébauche une politisation de sous-officiers et d'hommes du rang qui fera son chemin. (...). Les rumeurs qui couraient depuis des mois prennent corps : l'armée bouge"

62. Le Derg, en amharique, ou le collège.

63. Provisional Military Administrative Council, PMAC.

\section{RÉSUMÉS}

Having only been left to the initiative of the clergy till the end of the $19^{\text {th }}$ century, the first official schools were created under Melenik I's reign. This education system, together with a certain nomber of private schools set up by western religious orders, was to develop thanks to, in particular, the establishment of a Ministry of Education in 1930 by Haile Selassie. The momentum was interrupted between 1935 and 1941 with the Italian invasion but it picked up from then onwards with the introduction of a land tax aimed at financing the education system.

The first Conference of the African States' Education Ministers took place in Addis Abbeba in 1961 and the Unesco report presented there denounced the short-comings of the education system in Ethiopia : primary education only covered $5.6 \%$ of the children from the related age bands. Furthermore, education was restricted to the dominant ethnic group, in their language, Ahmaric, and clearly favoured cities over rural areas.

Once this situation was denounced, the Empire tried to boost the fortunes as a Modern State by implementing policies for literacy and for developing education.

Nevertheless, just before the 1974 military coup, the situation, increasingly denounced by students themselves, had hardly changed: $18 \%$ of children were in primary school in towns and only $1 \%$ in the countryside. 


\section{AUTEUR}

\section{ALAIN VERHAAGEN}

Faculté des Sciences sociales, politiques et économiques - Université Libre de Bruxelles Belgique 